\title{
Innovación constructiva a principios del siglo xx: Preámbulo a la modernidad arquitectónica y arquitectura subestimada
}

\author{
Early $20^{\text {th }}$ century building innovation: Architectural modernity's preamble \\ and under-estimated architecture
}

\section{$<$ Resumen>}

Las primeras décadas del siglo xx representan un período que adquiere singular importancia para la historia de la arquitectura chilena en general y particularmente para el caso de la ciudad de Santiago. Etapa de transición, que reproduce procesos gestados en otras realidades socioculturales es un campo de estudio que no ha sido analizado con el detalle que demanda, exaltándose principalmente, la modelística del Movimiento Moderno como causa y razón que precipita la modernidad arquitectónica local.

Sin embargo, existirían otros aspectos que, con un carácter rupturista menos evidente quizás, colaboraron igualmente en instaurar una modernidad histórico-arquitectónica en nuestro medio. Es el caso de la innovación técnica que de forma anticipada a la irrupción de la arquitectura moderna propiamente tal comenzó a presagiar, posibilitar y demandar el cambio modernizador que comenzaría a operar posteriormente. La introducción del hormigón armado, en tanto material y nueva concepción constructiva que su uso conllevaba, ejemplifica esta situación.

$<$ Abstract>

The first decades of the 20th century represent an especially important period in the history of Chilean architecture in general, particularly in the architecture of the country's capital city Santiago. It is a phase qualified as a transitional one, when avant garde foreign models that answer to different social and cultural realities were reproduced locally. This phenomenon has not been studied with the appropriate depth of analysis, and frequently the ideology and models of the so called Modern Movement are highlighted as the sole source that launched the local architectural modernity

Nevertheless, there are other factors that, even with a less evident revolutionary character, contributed significantly to the burst of historical and architectural modernity in the country. That is the case of the innovation in building technology that prior to the appearance of the modern architecture itself started to pave the way for make possible and demand the modernizing change to follow. The introduction of reinforced concrete, both as a material and as the new building conception that it implies, is a good example.

<PALABRAS CLAVE >

INNOVACIÓN TECNOLÓGICA / HORMIGÓN ARMADO

MODERNIDAD ARQUITECTÓNICA

$\angle K E Y W O R D S$,

TECHNOLOGICAL INNOVATION / REINFORCED CONCRETE I ARCHITECTURAL MODERNITY

\section{Introducción}

Teniendo en consideración la realidad sísmica del país, que desde principios de la instalación colonial se manifestó trágicamente con toda su acción destructora, la arquitectura chilena adoptó tempranamente un importante rigor constructivo, donde la condición estable de los edificios era continuamente puesta a prueba, transformándose en una preocupación primordial que muchas veces, incluso, limitó la inventiva arquitectónica. Junto con ello, la escasa disponibilidad de recursos materiales, como también técnicos y profesionales, instauró durante el período colonial un modo de proceder basado en un empirismo modelado por un proceso continuo de ensayo y error que, en definitiva, propició el establecimiento de soluciones constructivo-arquitectónicas convencionales.

Arquitecto Universidad de Chile, 1982. Académico Departamento de Historia y Teoría de la Arquitectura. Facultad de Arquitectura. Universidad de Chile. Profesor de Historia de la Arquitectura Chilena. 
Se puede por lo tanto entender, de ese modo, la condición tradicionalista que asumen muchas de las manifestaciones edificatorias locales durante aquel período y que se explicitan en el reducido repertorio tipológico arquitectónico colonial. De éstos, y a modo de ejemplo, el más representativo lo constituye la casa urbana colonial con su característica conformación que se mantiene casi inalterable a lo largo del tiempo.

Lo que ha sido caracterizado más arriba, quizás desde un punto de vista un tanto extremo, tiene por objetivo ilustrar de qué modo la práctica constructiva y arquitectónica estuvo, en gran medida, constreñida en su desarrollo durante la época colonial en cuestión, y no sólo por las carencias que han sido referidas, sino que por cierto inmovilismo operante que no favorecía la innovación tecnológica y proyectual. Prueba de ello es lo determinante que resultó ser, en ese sentido, la irrupción e influencia de funcionarios de la corona especialistas en el arte de la arquitectura y la ingeniería militar a partir de la segunda mitad del siglo xvIII que, con un dominio disciplinar más elaborado -académico y práctico- dio pie para iniciar una nueva etapa en la praxis arquitectónica. Lo mismo ocurrió a mediados del siglo XIX cuando, ya en el contexto histórico republicano, se incorporó nuevamente un número significativo de arquitectos y mano de obra especializada extranjera al medio local. En ambas ocasiones es posible reconocer avances técnicos que establecieron formas renovadas de proceder que ampliaron las posibilidades edificatorias.

Sin embargo, una constante que trasciende a lo largo de los siglos, independiente de las fases señaladas, es el hecho que durante todo este desarrollo histórico, las soluciones constructivas mantuvieron una concepción que privilegió el uso del muro como elemento protagónico primordial. Sea arquitectura de adobe o de ladrillo, sea en el siglo xvIII o en el xIx, fueron los muros los encargados no sólo de conformar los límites de los recintos en un edificio, sino que de constituir el sistema estructural encargado de asegurar las condiciones adecuadas de resistencia, rigidez y estabilidad. De ese modo se estableció un modelo normado por la capacidad mecánica de los muros en cuanto soporte estático con un compromiso indeclinable frente a las solicitaciones dinámicas originadas por la realidad sísmica local. Es esta última responsabilidad estructural, quizás, la que mejor explica la estereometría mural, la cual se modeló en la experiencia constructiva en fábricas de adobe la que le imprimió características, tales como: la restringida amplitud y proporción de los vanos respecto de la superficie del muro privilegiando la masa que asegure la inercia de éste; la limitada dimensión en el ancho de las crujías estructurales dado el uso de entramados de entrepiso y armaduras de techumbres resueltos en base a piezas de madera, de modo de no sobrecargar innecesariamente los muros de descarga y aminorar el efecto volcante; una planimetría compartimentada que permite un adecuado arriostramiento mediante el encuentro secuencial de muros perpendiculares a las líneas estructurales principales.

También debe mencionarse la limitación en la altura (esbeltez) de la estructura mural dada la inestabilidad latente frente a la fuerza volcante ocasionada por los sismos, aspecto que fue particularmente determinante en el período colonial, donde fueron escasas las construcciones que sobrepasaran la altura de un piso. Igualmente, el requisito de estabilidad edificatoria, condicionó el ancho de dichos muros, los cuales eran de espesores considerables.

Los procedimientos constructivos que han sido descritos, basados más bien en criterios operativos de origen empírico que perseguían la estabilidad de los edificios, concuerdan con lo que se encasilla como propios a una mentalidad precientífica. Es decir, resolver el desafío estructural edificatorio mediante técnicas tradicionales verificadas en la práctica constructiva que aseguren un comportamiento estático adecuado, y donde, precisamente, los edificios de fábrica son el mejor ejemplo de adaptación a dicha manera de operar. En ello sería determinante el que dichas estructuras trabajan a compresión evitando los esfuerzos de tracción, especialmente en las uniones.

Las consideraciones constructivo-estructurales que han sido descritas hasta este momento, aplicables al desarrollo general de la arquitectura santiaguina hasta fines del siglo xIx, han tenido por objeto caracteriza su condición tecnológica marcadamente tradicionalista (precientífica) como ya se ha señalado, por lo cual corresponde ahora revisar con especial interés lo ocurrido a partir de 1900 cuando comenzó a operar con mayor intensidad la innovación tecnológica en el ámbito arquitectónico local; ello queda demostrado por el hecho de ser el momento en que se lleva a cabo la introducción del uso del hormigón armado en la arquitectura de la ciudad de Santiago, material que, como ha sido enunciado, desempeñó un importante rol en propiciar la modernidad.
En ello no deja de ser significativo el hecho de que su uso presupone un conocimiento disciplinar especializado que se relaciona con la teoría del cálculo estructural que, en definitiva, cuestionará e innovará los criterios constructivo-proyectuales precedentes.

\section{El uso del hormigón a rmado e n la \\ arquitecturade Santi a go}

Primera fase

El año 1906 señala el inicio de la producción industrial de cemento en Chile, fecha que es cercana a la que constituiría, según algunos autores, la primera manifestación del uso del hormigón armado en el ámbito propiamente arquitectónico. Ello correspondería a una obra proyectada cerca de 1900 por el ingeniero y arquitecto francés, avecindado en Chile, Eugenio Joannon Croizier a quien, por lo tanto, se considera como uno de los que introdujeron el uso de este material en la arquitectura local.

«A principios de siglo, en la época que los ingenieros de la Cía. Holandesa de Construcciones construían con este material los cajones flotantes del dique de Talcahuano $y$, posteriormente, el puente de la calle Quillota en Viña del Mar -primeras obras hechas en Chile en hormigón armado- él lo empleaba en la Casa de las Hermanitas de los Pobres, en calle Carmen...». (Fresno, CA N 55, 1989).

El uso mencionado se refiere a que en dicho edificio, con muros de fábrica de ladrillo, los entrepisos habrían sido proyectados por Joannon, a nuestro parecer, con un sistema en que combinaba relleno de concreto de cemento entre las vigas de fierro tal como él mismo recomendaba hacerlo posteriormente, en 1903, cuando se le encargó la construcción de un edificio comercial, la Casa Prá. Por lo tanto, no es exactamente el concepto actual del hormigón armado, sino un sistema constructivo de fierro revestido de cemento que él mismo habría comenzado a utilizar persiguiendo «levantar construcciones a la vez resistentes a los temblores e indestructibles por los incendios. (...) Las torres de la iglesia de San Ignacio, construidas de esta manera, me dieron un espléndido resultado. El fierro que forma el esqueleto de la construcción i asegura su estabilidad está completamente revestido con cemento, lo que asegura su conservación indefinida». (Benavides, CA N 55, 1989). 


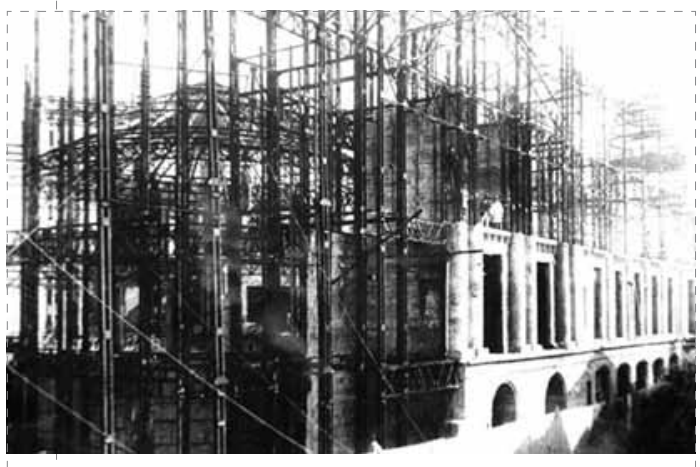

Edificio de la Bolsa de Comercio, Santiago ISeminario La Bolsa de Comercio. Santiago-Chile. Autor: Guillermo Pincheira, 1960 . En: Archivo de Arquitectura Chilena, FAU, Universidad de Chile.

No deja de ser ilustrativo lo que se ha descrito en la cita precedente. Es importante recalcar la formación europea y específicamente francesa de Joannon, en cuanto ingeniero y arquitecto, lo cual le permitía dominar de un conocimiento técnico de primera fuente respecto de los adelantos constructivos de la época y visualizar su posible aplicación en Chile, aspecto en el cual destacó. Debe mencionarse al respecto que dicho interés y experiencia profesional y su vinculación a la enseñanza universitaria le lleva a escribir y editar el libro EL PAPEL DEL ARquitecto en las construcciones de Cemento Armado. Hay que reconocer, sin embargo, que en base a lo que el mismo Joannon señala la técnica constructiva descrita obedece más bien a una indagación intuitiva que a un dominio teórico acabado, aunque no obstante dicha solución constructiva no se entendería sin que el arquitecto tuviera una mínima certeza respecto de su adecuado comportamiento estable. Esa convicción previa habla precisamente de una época en que ya se contaba con un dominio sobre la teoría de resistencia de materiales y que permite ciertas elucubraciones proyectuales

El caso de Joannon no fue excepcional, sino que representativo del camino que siguieron varios otros arquitectos de la época -preferentemente europeos o con estudios en ese continente- que igualmente innovaron en el ámbito de las técnicas constructivas locales. De hecho el sistema de esqueleto de perfiles de fierro revestido de concreto dio forma a una serie de edificios donde dicha solución constructiva se aplicó a la totalidad de la obra arquitectónica. Es el caso, por ejemplo, del edificio Gath y Chávez de los arquitectos Alberto Siegel (austríaco) y Augusto
Geiger (suizo) que fuera construido en 1909 (demolido), el Palacio Undurraga obra de arquitecto español José Forteza del año 1911 (demolido), o el edificio de la Bolsa de Comercio diseñado por el arquitecto francés nacido en Chile Emilio Jèquier, realizado en e año 1913.

Hay que señalar, sin embargo, que son obras que en su concepción general se mantienen apegadas a la tradición academicista en cuanto organización espacial y expresión formal, por lo cual, junto con el uso de perfiles en vez de barras de acero, se establece que corresponden a una primera fase en la evolución del uso del hormigón armado en la arquitectura de Santiago.

Reconocemos que establecer esta primera etapa puede resultar controversial ya que, si se acepta lo que el mismo Joannon señala, esto es que el esqueleto de acero es la estructura resistente, y el hormigón sólo un material de revestimiento, no correspondería en estricto rigor a la definición propiamente tal de lo que, desde el punto de vista de trabajo mecánico, se concibe por hormigón armado. Sin embargo, creemos que en la práctica el hormigón no asume sólo un mero rol complementario como asegura Joannon, sino al conformar parte del elemento constructivo igualmente, aunque así no haya sido contemplado, adquiere un compromiso estructural (estático), lo que ocasionaría que, en realidad, ambos materiales -los perfiles de acero, y el hormigón- tengan un comportamiento mecánico. Por otra parte, respecto del trabajo a la flexión, habría que considerar que al concentrarse el área de acero en el alma del elemento constructivo, la masa de hormigón más excéntrica, en principio, estaría expuesta a fisuración por efecto de tracciones por deformación (pandeo o flexión), pero, dada la rigidez que aporta la sección maciza de acero ese problema se reduce de forma sustancial siendo, incluso, despreciable. De ese modo consideramos que el término hormigón armado estaría bien aplicado, sólo que lo reconoceríamos así en una fase inmadura de diseño estructural, donde la armadura de acero -perfil macizoestá sobredimensionada y funcionalmente ma localizada.

\section{Segunda fase}

Una segunda fase sería cuando se comienza a incorporar en propiedad las armaduras de barras de acero. Según datos históricos e primer edificio de esas características habría sido construido alrededor de 1910 constituido por vigas, pilares, losas y muros de hormigón armado, empleando vigas invertidas, sistema novedoso conocido como «suizo» en la época. Correspondería a un edificio que se ubicaba en calle Alameda (Avda. del Libertador B. O'Higgins R.), a la altura del 1.700, cuyo autor fue el arquitecto chileno Alberto Cruz Montt (CHEUL y cols., 1971). Este profesional realizó sus estudios de arquitectura en París, en la Ecole Speciale d'Architecture titulándose en 1900, y donde con seguridad se familiarizó con esta nueva técnica constructiva. De hecho a este arquitecto le correspondió diseñar el primer edificio en altura que se construyó en Santiago en el cual se empleó el referido material (Edificio Ariztía, levantado en 1921 y que consta de 10 pisos destinados a oficinas, más dos pisos superiores a modo de remate esquinero). Anteriormente, en 1917, había proyectado el edificio del Club de la Unión y con posterioridad, en 1926, el edificio para el Banco Central, los que aunque menos atrevidos en cuanto tipo arquitectónico sobresalen por las proporciones con que los dotó Cruz Montt gracias al uso del hormigón armado.

Un caso similar es el Ricardo Larraín Bravo, chileno igualmente titulado en Francia en la Ecole Speciale d'Architecture y socio por un tiempo de Cruz Montt, quien tempranamente, en 1912, elabora el proyecto para una de las obras más ambiciosas de aquellos años; la iglesia de los Sacramentinos, obra que explota un uso volumétricamente más expresivo del material. También se podrían nombrar el Edificio Díaz, de 1925, obra de Valdivieso y de la Cruz que, al igual que el Ariztía, son los primeros rascacielos de la época; los edificios para el Banco de Londres (1918) y el Banco de Chile (1922), ambos de Siegel, o el edificio destinado a oficinas e imprenta del Diario llustrado proyectado por Manuel Cifuentes (1914), por citar aquellos casos más representativos.

Por otra parte, el nuevo material se fue haciendo más conocido no sólo a través del uso que de él se hacía, sino que se pueden reconocer iniciativas, en aquellos años, con el fin de divulgar su conocimiento y aplicación. Así, por ejemplo, se puede documentar la conferencia «El Concreto Armado. Nociones Generales», hecha en la Universidad Católica el 21 de Junio de 1912, bajo la presidencia del Exmo. Ministro de Francia señor Paul Veillet-Dufrèche. Estuvo dedicada al doctor Guillermo del Sol, por Víctor Auclair, arquitecto diplomado de la Escuela de Bellas Artes de París; premiado en los concursos Godebœuf y Rougevin, 1895 y 1896; alumno de MMs. Moyaux y Vaudremer, Membres de l'Institut, Directeurs Gènèraux de Bâtimentes civils de 


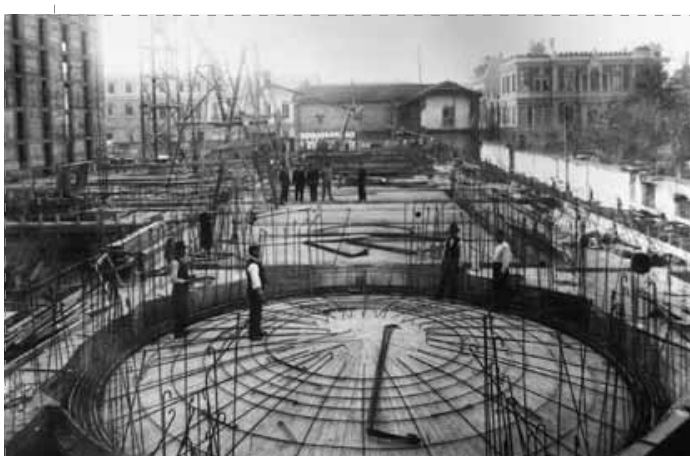

Biblioteca Nacional. Piso bajo volumen Museo Histórico actual Archivo Nacional. ISeminario Biblioteca Nacional. Autor: Mariana García, 1959. En: Archivo de Arquitectura Chilena, FAU, Universidad de Chile)

France. La citada conferencia fue reproducida posteriormente en el №2 (1913) y N5 (1915) de la revista De Arquitectura, publicación de la Sociedad Central de Arquitectos de Chile. Se señala como motivo de la venida del arquitecto Auclair a Chile su interés por verificar el uso del hormigón armado en construcción enfrentadas a la exigencia sísmica y se menciona la existencia de un Sistema Constructivo en Concreto Armado Victor Auclair. Este arquitecto participa en diferentes obras realizadas en Santiago en las primeras décadas del siglo xx, asumiendo su condición de especialista en el uso del hormigón armado (se le adjudica por ejemplo la construcción y quizás diseño de la marquesina del Club Hípico de Santiago). Igualmente, se establece que en el año 1914 comenzó a enseñarse la Teoría del Hormigón Armado en la Escuela de Arquitectura de la Universidad de Chile.

En todo caso, al igual que en la primera fase, hay que hacer notar que en los casos antes reseñados el sistema constructivo quedó opacado bajo el ropaje académico en el cual se habían formado sus creadores. Así resultaba difícil adivinar que tras zócalos almohadillados y fachadas de estilo se escondía un material constructivo revolucionario. Es esta condición, donde el material no es utilizado en forma honesta y en toda su potencialidad, imitando más bien las formas y tectónica de los sistemas constructivos tradicionales, lo que caracteriza esta segunda etapa evolutiva. Hay que reconocer, eso sí, el beneficio de un mayor alarde proyectual evidenciado en un manejo más monumental de las proporciones y la solución estructural, pero que, sin embargo, reafirma el discurso historicista. El caso que mejor ejemplifica esta fase del uso que se hacía del material lo constituye el edificio para la Biblioteca Nacional -en la mejor tradición del estilo beaux arts- aunque construido íntegramente en hormigón armado a partir del año 1919

\section{Tегсега fase}

La tercera fase se inicia cuando se produce una apropiación más efectiva del hormigón armado, al asumir ahora el desafío proyectual del material de forma madura, buscando aprovechar de mejor modo sus posibilidades constructivas y estructurales, y expresar adecuadamente su naturaleza. Es este último aspecto donde se manifestaba con mayor claridad, quizás, una suerte de crisis en la práctica arquitectónica dando pie a una autocrítica por parte de algunos arquitectos respecto del uso que se ha venido haciendo del hormigón armado. Es lo que expresa el texto escrito por el profesor León Jaussely (n. Toulouse, 1875 - m. Givry, 1933) ${ }^{[1]}$. La enseñanza de la Arquitectura en lo futuro, reproducido el año 1925 en el N 12 de la revista EL ARQUITECTO (órgano oficial de la Asociación de Arquitectos de Chile), cuando Chile está aprobando una Constitución Política que dará amplias atribuciones al Estado para conducir el desarrollo y dirigir los destinos de nuestra sociedad.

En este escrito el autor reflexiona acerca del principio ético que debe animar el desarrollo de la arquitectura en aquellos decisivos años, incentivando el compromiso con el espíritu de tiempo, lo cual se relaciona en gran medida con el reconocimiento hacia las nuevas técnicas y el uso honesto que de éstas se debía hacer. Igualmente, se insinúa de qué modo la estética empieza a comprenderse más bien ligada a la tectónica que da forma a un edificio más que a consideraciones meramente formal-estilísticas.

Un aspecto de distinta índole que también habría que considerar como antecedente que propicia el cambio en la concepción arquitectónica en aquellas décadas del siglo xx tiene que ver con la variable económica que comienza a ser cada vez más determinante en la instancia proyectual, en una época en que ya no se dispone de recursos como antaño, y demanda, por lo mismo, de mayor racionalidad y austeridad.
Es posible que la promoción de esta tercera fase del uso del hormigón armado en nuestro medio tenga relación, además, con dos hechos de distinta naturaleza que tienen lugar casi simultáneamente. El primero tiene que ver con la construcción el año 1929 del edificio Oberpaur que historiográficamente se considera el primer edificio moderno de la arquitectura santiaguina. Obra de los arquitectos nacionales Jorge Arteaga y Sergio Larraín García-Moreno, ésta fue la primera manifestación local en que se reconoce la influencia, casi textual, de las vanguardias europeas -concretamente del expresionismo alemán- que dio forma a un edificio de marcada racionalidad en su concepción y expresión formal expresada en el rigor constructivo con que se manifiesta el empleo del hormigón armado como material configurador del edificio. De ese modo, esta obra se transforma en una imagen patente de los nuevos caminos que se abrían a la arquitectura y de la expresión moderna del hormigón armado.

El segundo suceso tiene que ver con nuestra realidad sísmica, debido al hecho que el año 1928 tuvo lugar el gran terremoto que afectó principalmente a la provincia de Talca con un alto grado de destrucción y numerosos muertos. Ello generó un profundo cuestionamiento de la forma como se venía desarrollando la práctica constructiva en el país, dominada aún por sistemas tradicionales no controlados. Como resultado de ello, en el año 1929, se dictó la Ley 4.563 que constituye la primera iniciativa por regular la práctica constructiva en Chile:

«En este cuerpo legal encontramos e primer atisbo de ordenación urbanística de las ciudades, al disponer que aquéllas que tuviesen más de 20.000 habitantes deberían elaborar un plano general de transformación, que serviría para dar las líneas de edificación, además de contemplar la obligación de crear en la respectiva municipalidad el cargo de Director de Obras, con la exigencia habilitante para su desempeño de investir la calidad de ingeniero civil o de arquitecto. Especial mención merecen las facultades concedidas tanto al Presidente de la República, para dictar ordenanzas que regulen la construcción, como a las municipalidades, para otorgar los permisos de construcción en toda ciudad con más de 5.000 habitantes» (Cita extraída

[1] Ecole des Beaux-Arts de Toulouse, proyectista de una extensión de Barcelona en 1904, visitante en Argentina en 1926. 
de Reseña Histórica. Ordenanza General de Construcciones y Urbanización, sexta edición).

En relación con los aspectos tecnológicos se señala que con posterioridad al terremoto que afectó la región central chilena en 1928 el gobierno contrató a ingenieros franceses y holandeses que con sus aportes metodológicos cooperaron en la difusión del empleo del cálculo estructural. Consecuencia de esto fue promulgada una ordenanza que obligaba al uso de pilares y cadenas de hormigón armado en las construcciones de albañilería de ladrillos (Ortega y Hermosilla, 1996).

Algo similar, pero a menor escala, había ocurrido luego del terremoto que asoló a Valparaíso en 1906, cuando se decidió reemplazar la cal por cemento Portland en los morteros de pega de las albañilerías de ladrillo.

De ese modo se puede verificar que en este período se perfiló de manera más adecuada la utilidad y aceptación, con rango oficial, del hormigón armado que, por lo mismo, comenzó a tener una expresión más reconocible. De esta forma la construcción comienza a tener una mayor aceptación como disciplina especializada. Hay que señalar, sin embargo, que aún predominará el protagonismo del muro como elemento principal en la definición de la obra arquitectónica, sobre todo en lo que se refiere a los planos exteriores.

Fases posteriores se establecen en el momento en que se impone una tectónica que finalmente tiende a superar todo precepto constructivo tradicional y explora las nuevas posibilidades que el material posibilita, lo cual en gran medida demandaba readecuar la concepción arquitectónica. Es lo que Victor Auclair presagia tempranamente desde una visión formalista cuando decía:

«Un asunto importante para el uso de concreto armado, y su porvenir, en arquitectura, es su estética. Tocante a esto, nadie duda que, por su naturaleza especial y el modo de usarlo, merece estudio especial.

Toda composición artística se reduce al estudio de la mole y de los modelados a los cuales se acostumbra el ojo. En la satisfacción que sentimos en presencia de una obra arquitectural hay mucha parte de costumbre; y toda modificación, todo cambio brusco en las formas adoptadas, produce extrañeza, molestia y un temor que nos hace primero protestar, y luego analizar. La calma y la tranquilidad no vuelven sino cuando nos hemos cerciorado de que la estabilidad, que es la primera condición de toda obra de construcción, existe en realidad» (Auclair, 1915).

Por su parte un autor contemporáneo acotará respecto de la singularidad del nuevo material:

«Pero el hormigón armado presentaba cualidades excepcionales para adaptarse a todo tipo de comportamiento, pudiendo variar ad libitum la dimensión y la forma de los elementos, la cantidad y la posición de los hierros, consiguiendo, dócilmente, resolver sin soluciones de continuidad el paso de las estructuras verticales a las horizontales, es más, transformando toda la construcción en un sistema reticular estructurado, sin poner demasiado en evidencia las diferencias formales entre una articulación y un empotramiento, negando prácticamente el simple apoyo y aboliendo de hecho la distinción entre elementos verticales y horizontales» (Quaroni, 1987).

La cita de Auclair resulta ilustrativa, dado que refuerza lo que ya ha sido señalado en el sentido de que la tradición academicista, imperante aún a principios del siglo xx, determinaba una forma de construir regulada por proporciones establecidas que regían no sólo la concepción (composición) global de una obra arquitectónica sino que aseguraban, además, su estabilidad de acuerdo al conocimiento empírico heredado basado igualmente en reglas de proporción geométrica aplicadas a los sistemas constructivos tradicionales. Del mismo modo, las observaciones de dicho autor insinúan el principio innovador que presagiaba el uso del hormigón armado que precisamente cuestionará las convenciones constructivas y desplazará, en definitiva, las reglas de proporción tradicionales como criterio de estabilidad.

De esta manera, se podría afirmar que la aceptación plena del hormigón armado en cuanto nuevo modo de construir demandaba de un proceso de asimilación y maduración que explica las etapas que se han señalado. Es por lo tanto válido plantear la idea de que en las primeras décadas del siglo xx la arquitectura local no habría estado preparada aún para desligarse de ciertas convenciones constructivas, como por ejemplo, el rol protagónico asignado al muro en cuanto elemento definitorio en un edificio. Dada la conciencia sísmica local, tendrá que pasar un tiempo para que, gracias a la asimilación gradual de la estética de la modernidad y su ideología, y a un rigor más evolucionado respecto de la verificación matemática del dimensionamiento y capacidad resistente de os elementos estructurales, se logre modificar el gusto y, consecuentemente, se acepten concepciones estructurales más atrevidas.

Al respecto cabe destacar que diez años después de haberse dictado la primera Ordenanza General sobre Construcciones y Urbanización tal como lo establecía la Ley General del año 1929, fue designada una comisión para revisar dicha Ordenanza a consecuencias de un nuevo terremoto ocurrido en Chillán (Decretos 322, de 15 de febrero y 651 de 11 de abril de 1939, Ministerio de Fomento). Esta comisión elaboró un «Procedimiento aproximado para la determinación de la acción de los temblores en las construcciones», el que fue incorporado como Anexo II dentro de las modificaciones realizadas a la Ordenanza con fecha 10 de noviembre de 1949. Esta aproximación estará enriquecida por los conocimientos de diversos expertos de fama mundial que estudiaron los terremotos de San Francisco (1906), de Uraga (1922), de Tokio (1923), de Long Beach (1933). Entre ellos se puede citar a los profesores Harry A. Williams de la Universidad de Stanford, California (1937); el Prof. Omori (1928) quien había estudiado los efectos del terremoto de San Francisco de 1906 y del de Tokio de 1923 -donde según sabemos el único edificio que quedó intacto fue el Hotel Imperial de Frank Lloyd Wright-; el Prof. John R. Freeman, quien también había estudiado los efectos del terremoto de Tokio de 1923; el Prof. Imamura, de la Academia Imperial Japonesa y profesor de Sismología de la Universidad Imperial de Tokio; Ios profesores Leander M. Hoskins, John D. Galloway, y Lydik S. Jacobsen (1938) (INDITECNOR, 1949). EI informe citado revisa críticamente el método de cálculo de construcciones antisísmicas y en sus puntos 8,9 y 10 propone el método de cálculo de resistencia sísmica de tres tipos de estructuras de hormigón armado (muro, cepas de un puente y edificio de tres pisos). Lo expuesto demuestra cómo el hormigón armado, luego de cuatro décadas de uso, finalmente se ha impuesto como sistema constructivo avalado por su condición de material moderno, asegurando en alto grado la estabilidad de las edificaciones mediante el cálculo estructural.

De acuerdo a lo expuesto en relación al proceso de innovación tecnológica que se verifica en la arquitectura santiaguina, iniciadas las primeras décadas del siglo xx, es posible reconocer cómo la componente técnica fue asumiendo, progresivamente, una mayor consideración proyectual que tendió a restituir el equilibrio de variables 
que subyacen tras el hecho arquitectónico (utilitas, firmitas y venustas, siguiendo el discurso vitruviano). Así se puede verificar una actitud renovada respecto del modo de encarar la obra de arquitectura -reflejo del espíritu del tiempo- la cual debe considerarse expresión de una voluntad modernizante en el sentido que promovió la superación de la etapa histórica precedente, caracterizada por preocupaciones marcadamente formales y prácticas constructivas tradicionales. Dicha nueva actitud, a su vez, se fundó y benefició directamente de la asimilación y difusión progresiva del uso del hormigón armado en el ámbito edificatorio.

La incorporación de este nuevo material en la escena local demandó readecuar el modo de concebir y enfrentar la práctica constructiva en la medida que su uso obligaba asimilar un conocimiento teórico esencial - de carácter científico- relacionado con el comportamiento tensional de los materiales. Ello tuvo como consecuencia inmediata el poder reemplazar la intuición de ascendencia meramente empírica por certezas fundadas en predicciones matemáticas respecto de cómo diseñar la estructura de un edificio en función de las demandas de resistencia y estabilidad, pudiéndose incluso, asegurar su desempeño futuro. Por ello mismo, era del dominio de dicho conocimiento teórico de lo que dependía, en definitiva, el provecho que los arquitectos del período pudiesen ser capaces de obtener del hormigón armado e iniciar, en lo posible, una nueva etapa históricoarquitectónica.

Igualmente, dada la condición sísmica del país su introducción resultó del todo oportuna, si se tiene en cuenta la capacidad de este material para resolver de mejor forma las muchas veces extremas solicitaciones tensionales a que se ven sometidas las estructuras arquitectónicas en el medio local donde, por lo mismo, la condición estable es determinante para la seguridad edificatoria.

Es precisamente ante este desafío estructura donde el hormigón armado aportará su mayor potencialidad mecánica, dando inicio a un proceso de renovación indiscutible del diseño estructural-arquitectónico, el que ahora ya no estará tan condicionado por la disposición geométrica de los componentes estructurales, sino que la problemática principal consistirá, más bien, en determinar el correcto dimensionamiento de éstos. Es este aspecto por lo tanto lo que posibilitó que comenzara a manifestarse un mayor grado de libertad en las concepciones arquitectónicas.
Asimismo, la condición de material artificial que singulariza al hormigón armado, posibilitando por lo mismo la manipulación interna de sus componentes -disposición y área de las barras de acero en conformidad a las exigencias tensionales- determina que el concepto mismo de dimensionamiento implique algo más que la simple apariencia morfológica externa de los elementos estructurales, ampliando y complejizando las posibilidades del diseño estructural.

De acuerdo a los alcances expuestos es comprensible que la incorporación de la tecnología del hormigón armado demandó una adecuación importante de la mentalidad constructiva de la época, determinando un proceso de asimilación paulatino e implicando, además, la consolidación del cálculo estructural como una especialización en el ámbito de la ingeniería civil.

Es en este contexto histórico-tecnológico inicial que se reconoce el desarrollo de ciertas manifestaciones arquitectónicas que, sin inscribirse necesariamente en la esfera de la arquitectura moderna, aportaron en el camino hacia la modernidad arquitectónica a modo de una indagación proyectual animada por el espíritu de época de innovación tecnológica y que temporalmente se ubicaría en lo que se ha denominado tercera fase de la incorporación constructiva del hormigón armado en la arquitectura de Santiago. Dentro de dicha etapa se vislumbra una transición conducente a una mayor consideración del diseño estructural en las obras arquitectónicas que tiende a restablecer la configuración constructiva como instancia proyectual reconocible.

Dichas manifestaciones arquitectónicas cabrían en la denominación de un protoracionalismo o racionalismo temprano, el cual no alcanza aún la estricta observancia funcionalista y rechazo absoluto a la reminiscencia academicista que supondrá posteriormente la instauración propiamente tal del Movimiento Moderno. Son, por lo mismo, obras arquitectónicas que suelen quedar opacadas por el desenfado de la vanguardia moderna, pasando muchas de las veces desapercibidas a pesar de sus méritos arquitectónicos, desconociéndose su contribución al progreso de la modernidad arquitectónica.

\section{Un caso гергеsentativo El edificio de la Caja Nacional de Ahorros}

Ubicado en la calle Morandé $N^{\circ} 147$, en la vereda oriente frente a la Plaza de la Constitución, fue construido en 1930, según refería una placa adosada al costado del acceso principal. Su autor fue el arquitecto chileno Ricardo González Cortés (n.1887, Santiago / m. 1957, San Vicente de TaguaTagua)

Su demolición hace unos pocos años atrás (año 2002), a pesar de estar inserto en la zona de protección patrimonial en torno al Palacio de La Moneda, es un claro ejemplo de la vulnerabilidad de las manifestaciones arquitectónicas de esta etapa del desarrollo urbano-arquitectónico local.

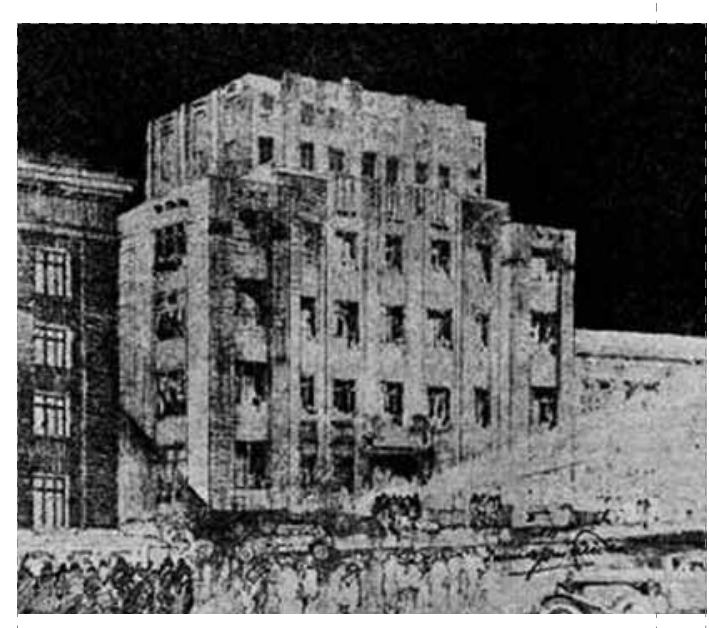

Revista Arquitectura y Arte Decorativo No 1 Año 2

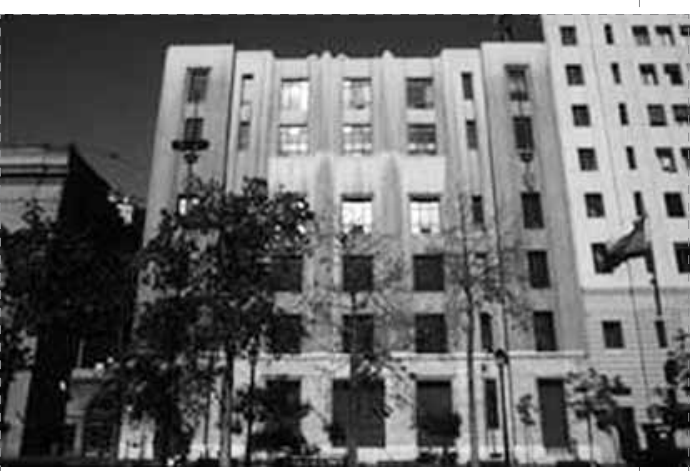

Archivo del autor. 
Revista Arquitectura y Arte Decorativo $N^{\circ} 1$ Año 2
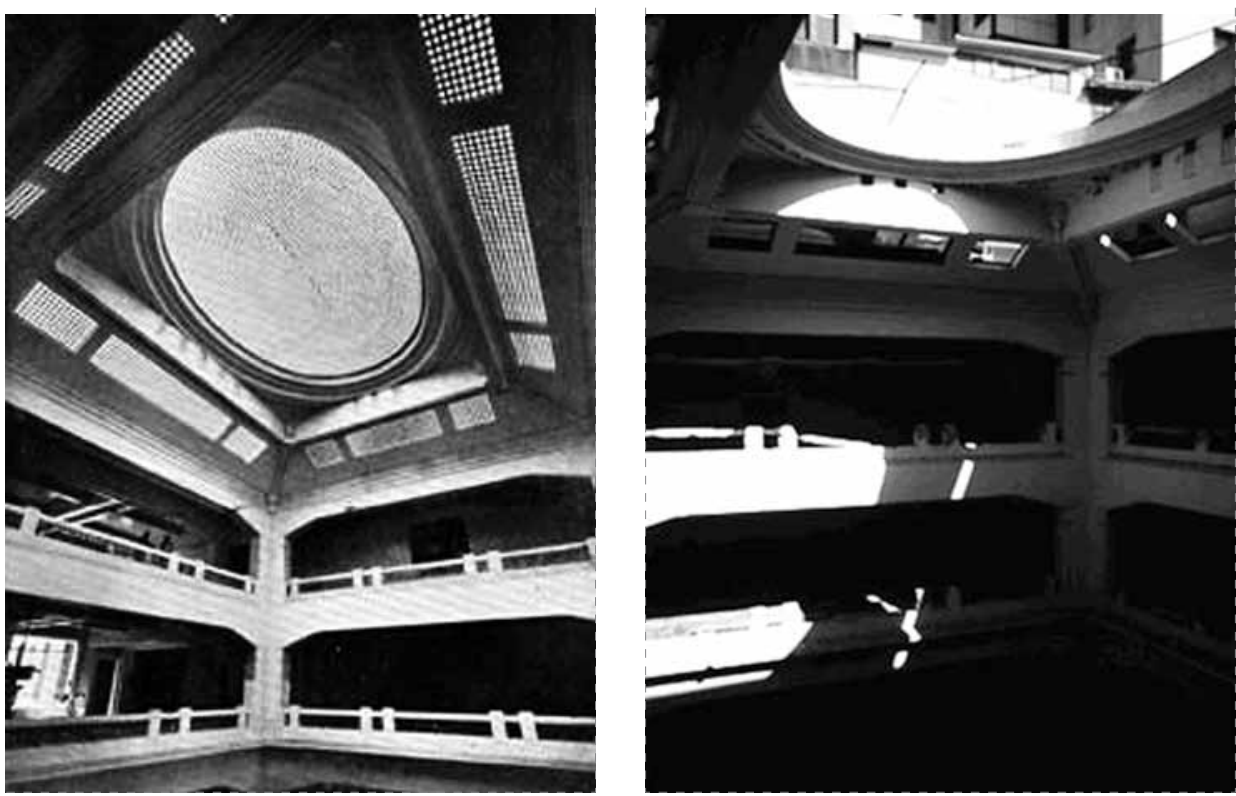

Sin ser una obra de la arquitectura moderna propiamente tal, corresponde a una obra de transición con innegables innovaciones en el ámbito constructivo y, por lo tanto, documentando y representando de forma ejemplar el avance disciplinar en pos de la modernidad arquitectónica local desde la indagación proyectual desde la componente técnica.

Las imágenes presentadas testimonian el espíritu innovador constructivo que animaba este ejemplo de la arquitectura de esos años aprovechando claramente las posibilidades modernas que inauguraba el hormigón armado. Era posible apreciar, incluso, cierto grado de alarde estructural evidente que difícilmente puede pasar desapercibido y que verifica el hecho ya señalado de que, adelantándose a la consolidación de la forma arquitectónica moderna, la variable técnica empezó a jugar un rol precursor significativo y no muchas veces destacado por la historiografía arquitectónica. Se debe mencionar además el hecho significativo de que este edificio al igual que otros edificios similares y contemporáneos fue capaz de resistir exitosamente el terremoto de Santiago de marzo de 1985 que sin duda fue una prueba altamente exigente para la arquitectura de muestra ciudad. Ello demuestra entonces como el nuevo sistema constructivo fue correctamente aplicado inaugurando una nueva época para la arquitectura local desde las condición resistente. Paradójicamente, sin embargo, el haber resistido exitosamente el embate sísmico más importante de la segunda mitad de siglo xx no «lo salvó de la picota» alentada por los intereses de la especulación inmobiliaria y de la ignorancia de quienes no supieron reconocer sus méritos y defenderlo en su momento.

\section{Bibliografía}

Allen, E. «Cómo funciona un edificio». Editorial Gustavo Gili, Barcelona, 1993. $4^{\mathrm{a}}$ edición. Título original: How Buildings Work. The Natural Order of Architecture, 1980

Auclair, V. «El Concreto Armado. Nociones Generales», en: Revista de Arquitectura, Publicación de la Sociedad Central de Arquitectos de Chile, $\mathrm{N}^{\circ} 2$, pp. 23-24, Santiago, 1913 y N 5, pp. 57-59, Santiago, 1915.

Aroca, R. «Arriostramiento». Separata. Cuadernos del Instituto Juan de Herrera de la Escuela de Arquitectura de Madrid. Apunte docente del curso Proyecto de Estructuras del Programa de Doctorado en Arquitectura y Urbanismo, Universidad de Chile Universidad Politécnica de Madrid. Santiago, 2002.

Benavides, J. «Un ecléctico con vocación técnica», en: CA N ${ }^{\circ} 5$. Revista oficial del colegio de arquitectos de Chile, pp. 67-71, Santiago, 1989

Cervera, J. «Diseño de Estructuras en edificación». Separata. Departamento de Estructuras de Edificación. Escuela Técnica Superior de Arquitectura de Madrid. Instituto Juan de Herrera, Madrid, 1993. Apunte docente del curso Proyecto de Estructuras del Programa de Doctorado en Arquitectura y Urbanismo, Universidad de ChileUniversidad Politécnica de Madrid. Santiago, 2002.

Cheul, R. y Arentsen, C. «Evolución histórica del hormigón armado en Santiago». Seminario de Investigación de Historia de la Arquitectura. Profesor guía: Raúl Farrú A. Facultad de Arquitectura de la Universidad de Chile, Santiago de Chile, 1971.

Fresno, L. «Eugenio Joannon Croizier. Biografía de una transferencia arquitectónica», en: CA № 55. Revista

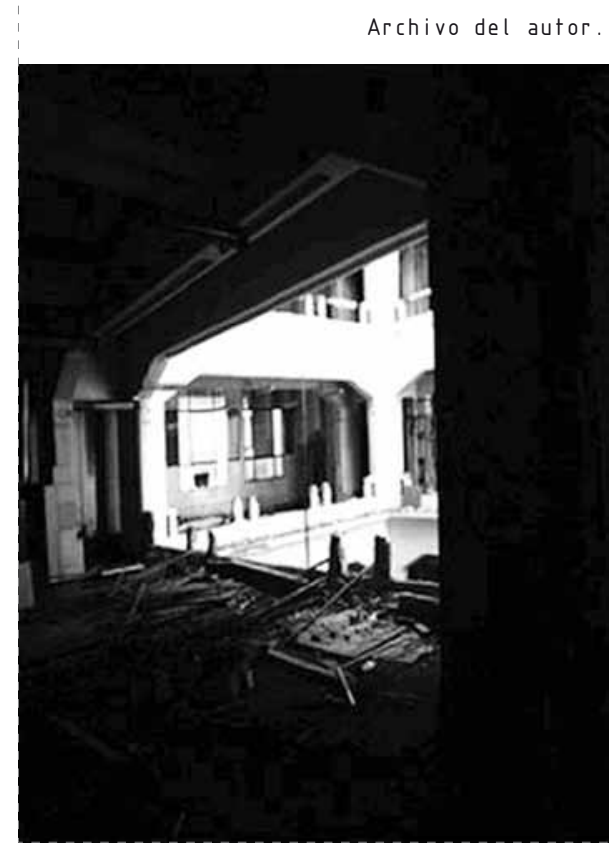

ficial del colegio de arquitectos de Chile, pp. 35-39, Santiago, 1989

González, J.L. y otro. «Claves del construir

arquitectónico. Tomo I. Principios». Editorial Gustavo Gili, Barcelona, 1997.

Gordon, J.E. «Estructuras o por qué las cosas no se caen». Celeste Ediciones, Madrid, 1999. Título original: Structures or Why thigs don't fall down 1978

Instituto Nacional De Investigaciones Tecnologicas Y Normalización, INDITECNOR, «Informe de la Comisión designada por Decretos 322, de 15 de febrero y 651 de 11 de abril de 1939, del Ministerio de Fomento, para estudiar el terremoto de Chillán». En: C.D.U. 69.05 + 711, 10 de nov. de 1949, Ley y Ordenanza General sobre Construcciones y Urbanización; pp. 73-84.

Jaussely, L. «La enseñanza de la arquitectura en lo futuro», en: El Arquitecto, № 12, Órgano oficial de la Asociación de Arquitectos, sin número de páginas, Santiago, 1925

Joannon, E. «El papel del arquitecto en las construcciones de cemento armado», en: CA N ${ }^{\circ} 55$. Revista oficial del colegio de arquitectos de Chile, pp. 35-39, Santiago, 1989.

Mas-Guindal, A. «Apuntes del curso Proyecto de Estructuras», correspondientes al programa de Doctorado en Arquitectura y Urbanismo, Universidad de Chile - Universidad Politécnica de Madrid, Santiago, Julio 2002.

Ortega, O. y Hermosilla, P.»|ntroducción de la edificación en altura en Chile», en: Revista De Arquitectura N7, Facultad de Arquitectura y Urbanismo, Universidad de Chile, pp. 14-21, Santiago, 1996.

Quaroni, L. «Proyectar un edificio. Ocho lecciones de arquitectura». Xarait Ediciones S.A., Madrid, 1987; original en italiano Progettare un edificio. Otto lezion di architettura, 1977

Torroja, E. «Razón y Ser de los Tipos Estructurales». Instituto Eduardo Torroja de la construcción y del cemento, Madrid, 1960. Tercera edición. 